\title{
Evaluation of life quality associated with health of girls aged 13-14 in groups with menstrual disorders and no disorders in health and development in the light of the questionnaire KIDSCREEN-52
}

\author{
Ewa Zięba ${ }^{1}$, Małgorzata Markowska², Jadwiga Krawczyńska³, Paulina Zięba" ${ }^{4}$ Grażyna Nowak-Starz ${ }^{1}$ \\ ${ }^{1}$ Developmental Age Research Department, Institute of Public Health, Faculty of Health Sciences, Jan Kochanowski University, Kielce, \\ Poland \\ Head of Department: Prof. JKU Grażyna Nowak-Starz \\ ${ }^{2}$ Department of Pathobiomechanics, Institute of Physiotherapy, Faculty of Health Sciences, Jan Kochanowski University, Kielce, Poland \\ Head of Department: Prof. Andrzej Rydzewski \\ ${ }^{3}$ Department of Oncology and Cancer Nursing, Institute of Nursing and Midwifery, Faculty of Health Sciences, Jan Kochanowski \\ University, Kielce, Poland \\ Head of Department: Prof. JKU Maciej Kielar \\ ${ }^{4}$ Jan Kochanowski University Graduate in Physiotherapy, Faculty of Health Sciences, Jan Kochanowski University, Kielce, Poland \\ Head of Department: Prof. JKU Grażyna Nowak-Starz
}

Key words: health related quality of life, maturation, menstrual disorders.

\begin{abstract}
Introduction: Quality of life is a multidimensional and interdisciplinary concept, combining a number of important areas of life. In terms of sociology, it is the degree of satisfying the essential human needs, while in terms of psychology it is psychological well-being. The most common understanding of life quality, accepted by the World Health Organization, is as follows: quality of life is how individuals perceive their status in life in terms of culture and value system in which they live and in relation to their goals, expectations, standards and interests.

Aim of the research: To compare two groups of girls, aged 13-14, with menstrual disorders and no disorders in health and development from urban and rural environments in the light of the questionnaire KIDSCREEN-52 with respect to individual dimensions.

Material and methods: We examined 122 girls with menstrual disorders and 240 girls without any disorders in health and development from primary and lower secondary schools from urban and rural environments. The study included girls attending primary and lower secondary schools at the age of 13-14. In the study we applied the standardized tool KIDSCREEN-52 Health Related Quality of Life Questionnaire for Children and Young People, in the Polish version of the questionnaire, to test the quality of life related to health of children and adolescents.

Results: Lower results with respect to all dimensions were obtained in the group of girls with menstrual disorders compared to girls without disorders in health and development in particular dimensions of the questionnaire KIDSCREEN-52.

Conclusions: Dissemination of activities which raise the quality of life, with particular emphasis on girls with menstrual disorders, is likely to affect significantly the health and quality of life in the future.
\end{abstract}

\section{Introduction}

In recent years the concept of quality of life has become one of the leading categories in Polish economics, humanities, social sciences and medicine. The term quality of life has no universally applicable meaning. It depends on a wider research approach and can be understood in various ways. One can state only that all the studies on the subjective perception of quality of life include the indicator of subjective "good life" or well-being experienced as satisfaction and happiness [1].

Quality of life is determined by human needs, beliefs, and values. It is a category variable in time and space, and it is a very personal feeling, individualized, and for different people has a different meaning. The effects of an objective situation in the evaluation of the quality of life in a given context may be completely different from the effects of the same situation in a different context. In the culture of ancient China the concept of quality of life was expressed in art, literature, philosophy and medicine. Maintaining a high quality of life is possible when Yin and Yang remain in harmony with each other. Man seeks in life material welfare and spiritual wealth [2].

Quality of life is a multidimensional concept, interdisciplinary, combining a number of important areas of life [3]. In sociological terms, it is the degree to which important human needs are satisfied, while in terms of psychology it means mental well-being [4]. 
With the emergence of the concept of life quality there appeared also the question of its assessment. Rating a particular part of life applies to everything that happens between an individual and the external and internal environment. Thus, in assessing the quality of life two elements can be distinguished: the objective situation in life, based on a factual database, and its subjective perception, based on the experiences and views of the individual [5].

"Quality of life evaluated objectively is a set of conditions of human life, the objective attributes of the areas of life, natural environment, objective health condition, the attributes of the material and the socio-cultural world" [6]. Studies on the overall sense of life quality provide evidence that objective factors explain only part of the experienced satisfaction. To a large extent the perception of overall well-being is attributed to the subjective quality of life, i.e. cognitive and emotional appraisal of particular aspects of life, and life as a whole, reflected in the level of general life satisfaction [7].

The concept of life quality refers to all aspects of well-being of an individual - both physical and psychological, and social, including important features of living standards, as well as the environment in which the individual lives [8].

The above-mentioned aspect is also important with respect to children. The International Convention on the Rights of the Child recognizes the right of the child to "the highest attainable standard of health, leisure and education. It also acknowledges the right to an adequate standard of living, to physical, mental, spiritual, moral or social development" [9].

All these aspects constitute appropriate criteria for life quality. The assessment of quality of life in children and adolescents developed in the European countries and the United States in the nineteen eighties and nineties. Up to the present new instruments for measuring the quality of life in children have been created. Quality of life is a particularly important dimension in the study of children with health problems. The problem of quality of life and its measurement has therefore become one of the crucial issues in paediatric practice [10].

Following the World Health Organization, the most common understanding of the quality of life is 'individuals' perception of their position in life in the context of the culture and value systems in which they live and in relation to their goals, expectations, standards, and concerns". In a broader sense quality of life is affected in a comprehensive way by physical health of individuals, their mental condition, independence level, social relationships, and personal beliefs with respect to the essential characteristics of the environment. The WHO definition also includes the cultural, social and environmental context. The concept of life quality developed out of, or in addition to, other terms, such as functional status, health status, functional disability, and life standards, adaptation or well-being [11].

\section{Aim of the research}

The aim of the study is to compare two groups of girls from urban and rural environments, aged 13-14, in the light of the questionnaire KIDSCREEN-52 with respect to individual dimensions. One group has menstrual disorders and the other has no disorders in health and development.

\section{Material and methods}

We examined 122 girls with menstrual disorders and 240 girls without any disorders in health and development. The girls are from primary and lower secondary schools from urban and rural environments. The first stage was the selection of the target group of girls with menstrual disorders. The girls in this group are patients of the Public Gynaecological Clinic at the Hospital for Gynaecology and Obstetrics in Kielce. Girls without menstrual disorders in health and development were selected as a comparison group. In the second stage two primary schools and two lower secondary schools were drawn, then the draw was made within the class.

A total of 14 classes were drawn; 7 classes of each type of school and girls with menstruation were selected from each class. The study included girls, aged 13-14, attending primary and secondary schools. The examined girls came from both rural and urban areas. We chose the method of team sampling with stratification based on the type of school and class. On the basis of data from the local education authorities, a list of primary and lower secondary schools located in the Świętokrzyskie Province was made.

The studies used the standardized KIDSCREEN-52 Health Related Quality of Life Questionnaire for Children and Young People in the Polish version of the questionnaire to test the quality of life related to health of children and adolescents.

\section{Results}

The results are representative of groups of girls during adolescence, aged 13-14, with diverse backgrounds.

Figure 1 shows the raw standardized results using the scale of 20-100 points and the results according to the standard ten scale for ten dimensions of the questionnaire KIDSCREEN-52 obtained from girls without menstrual disorders in health and development and girls, aged 13-14, with menstrual disorders from the urban area.

The results converted into the standard ten scale clearly indicate to what extent the quality of life in health of the examined girls differs from the average 


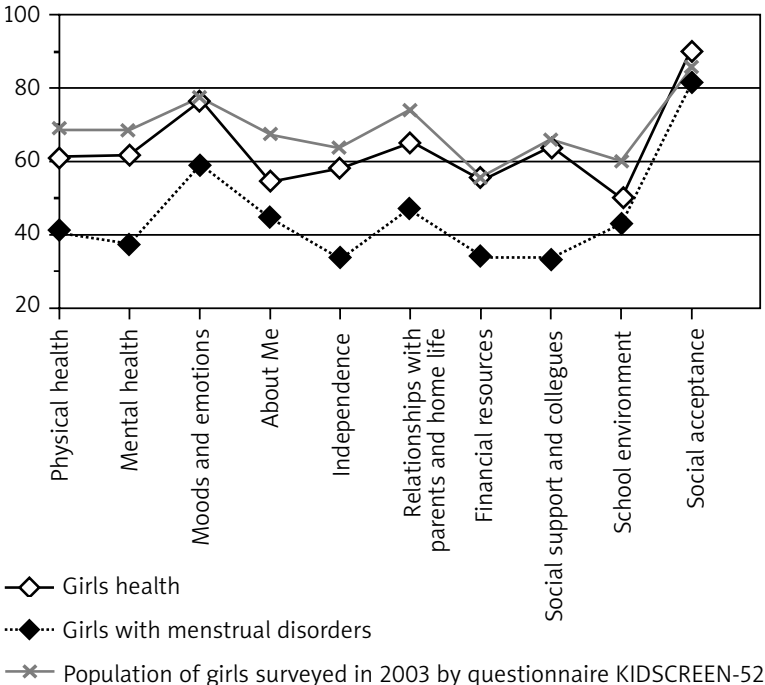

Figure 1. Profile of life quality related to health of girls without disorders in health and development and girls, aged 13-14, with menstrual disorders from the urban environment

studied population of girls in 2003 according to the questionnaire KIDSCREEN-52.

The studies were carried out by The Institute of Mother and Child in 2003 in order to develop its Polish version, which could be regarded as a kind of reference group - the standard. It should be noted that the test is promoted by the WHO and for each European country an appropriate language version of the test was prepared. In Poland this task was entrusted to the Institute of Mother and Child in Warsaw.

The analysis of the two groups indicates a better assessment of life quality in health by girls without menstrual disorders in health and development with respect to physical health, psychological well-being, moods and emotions, perception of oneself, independence and a better quality of life evaluation in health in terms of relationship with parents and family life, financial resources, and peer contacts. In terms of the other two dimensions - school environment and social acceptance - the difference between the examined girls is inconsiderable.

The comparison of indices in both groups of respondents to the study of girls aged 13-14 in 2003 according to the questionnaire KIDSCREEN-52 showed that girls with menstrual disorders are far below the standards of the 2003 respondents in the following dimensions: physical health, psychological well-being, moods and emotions, perception of oneself, independence, relations with parents and family life, financial resources, contacts with peers and school environment. In girls with menstrual disorders the closest dimension to the population of girls in 2003 is social acceptance.

The results of girls without menstrual disorders in health and development in terms of physical health,

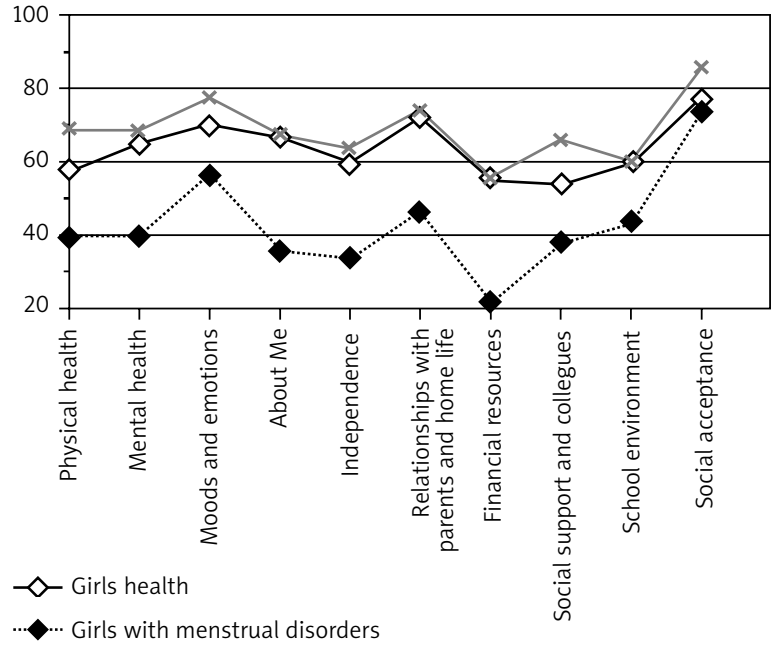

$\rightarrow$ Population of girls surveyed in 2003 by questionnaire KIDSCREEN-52

Figure 2. The profile of life quality related to health of girls, aged 13-14, without disorders in health or development and girls with menstrual disorders from rural areas

psychological well-being, self-perception, independence and in relations with parents, family life and the school environment slightly differ from the results of the population of girls aged 13-14 in 2003 according to the questionnaire KIDSCREEN-52. With respect to moods and emotions, financial resources and social support the results are in line with the standards of the population of girls in 2003. With respect to social acceptance the results for girls without menstrual disorders slightly exceed the standards of the population of girls aged 13-14 in 2003 in the light of the questionnaire KIDSCREEN-52.

The discriminating variable, which was the place of residence (rural area), was taken into account in the following analyses. Figure 2 shows the raw standardized results on a scale of 20-100 points and the results on a standard ten scale for ten dimensions of the questionnaire KIDSCREEN-52. The results were obtained from girls without menstrual disorders in health and development and girls, aged 13-14, with menstrual disorders from rural areas. The results converted into the ten standard scale clearly indicate how quality of life in health in the examined girls differs from the average of the studied population of girls in 2003 according to the questionnaire KIDSCREEN-52.

The analysis of the two groups indicates a better assessment of life quality in health by girls without menstrual disorders in health and development with respect to physical health, psychological well-being, moods and emotions, self-perception, independence and relationships with parents and family life, peer contacts as well as school environment.

In terms of social acceptance there is a slight difference between the examined girl. In girls, aged 1314 , with menstrual disorders from rural areas it is the 


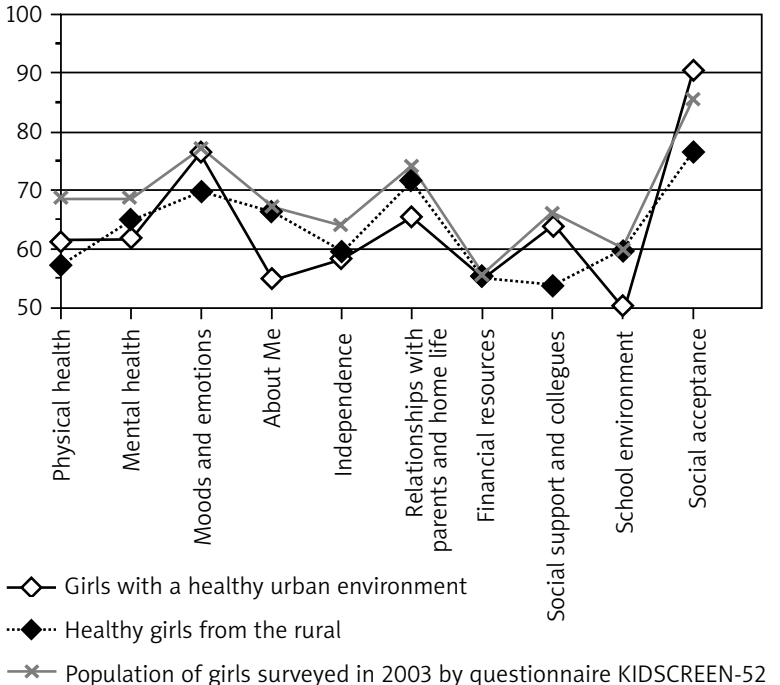

Figure 3. Profile of life quality related to health of girls, aged 13-14, without menstrual disorders in health and development from urban and rural areas

financial resources that attract attention. A very low level was noted, differing from the results obtained from girls without menstrual disorders in health and development.

The comparison of indices in both groups of respondents to the study of girls aged 13-14 in 2003 by the KIDSCREEN-52 questionnaire showed that girls with menstrual disorders are definitely below the standards of the examined girls in 2003 with respect to physical health, psychological well-being, moods and emotions, self-perception and independence, and peer contacts, as well as school environment and social acceptance.

The girls without menstrual disorders in health and development with respect to physical health, psychological well-being, moods and emotions, independence, peer contacts and social acceptance differ slightly from the results of the study on the population of girls aged 13-14 in 2003 according to the questionnaire KIDSCREEN-52 and are below the standard. With respect to self-perception, relations with parents and family life, financial resources and school environment, the results of girls without menstrual disorders correspond to the standards of the population of girls in 2003 in the light of the questionnaire KIDSCREEN-52.

The comparison of the two groups of girls, aged 13-14, from the urban and rural environment without menstrual disorders in health and development indicates a better assessment of the quality of life in health by girls from the rural area with respect to psychological well-being, self-perception, independence, relations with parents and family life and the school environment. Physical health, moods and emotions, social support among peers and social acceptance

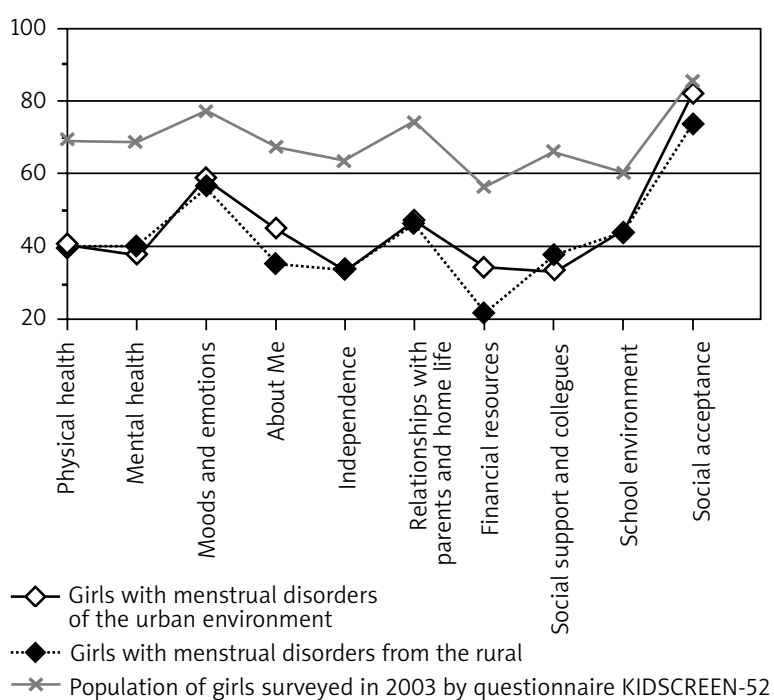

Figure 4. Profile of life quality related to health of girls aged 13-14 with menstrual disorders from urban and rural areas

are the remaining dimensions of the questionnaire KIDSCREEN-52 in which there were noted definitely better results in girls from the urban environment. With respect to financial resources the results are the same in both groups.

The comparison of indices in both groups of respondents with the study of girls aged 13-14 in 2003 according to the questionnaire KIDSCREEN-52 indicates that with respect to self-perception and school environment the girls from urban areas are significantly below the standard population in 2003. The other dimensions in both groups of girls are close to the above-mentioned norm.

The comparison of the two groups of girls aged 13-14 with menstrual disorders from urban and rural environments shows a gradual decline in the quality of life linked to health in relation to the studied population of girls in 2003 according to the questionnaire KIDSCREEN-52. The increase of social acceptance, where average indices of life quality increase, is the exception, while the average indices for financial resources decrease. The other dimensions of the questionnaire KIDSCREEN-52 are negligible with a tendency to higher values in girls from the urban environment.

\section{Discussion}

The importance of research on the health-related quality of life (HRQL) has increased in recent years. HRQL measures are now treated as outcome variables in many studies, and also constitute an evaluation criterion for intervention action. Life quality survey is a rapidly growing discipline that entered health sciences at a time when the need for new health indica- 
tors appeared. In Poland the professional application of questionnaires relating to quality of life of children and adolescents is experimental and is in the initial phase [12].

The KIDSCREEN project was implemented in 13 European countries, including Poland in the years 2003-2004, within the Fifth Framework Programme of the EU. As a result of its implementation six questionnaires were created to examine the quality of life related to health of children and adolescents aged 8 to 18 . The standardized questionnaire KIDSCREEN meets all the criteria that allow for monitoring the health of children and young people in the country and comparing it with other countries. At the survey stage risk factors and protective factors for health are examined. The relationship between health status, lifestyle and socio-economic situation of girls and their families and the indicators of life quality according to the questionnaire KIDSCREEN are shown.

The participation of Poland and other countries in the project was linked to the psychometric assessment of the questionnaire and adaptation of the instruments to the conditions of a particular country. KIDSCREEN-52 proved to be accurate and sensitive. The tool allowed us to assess the quality of life dimensions: physical health, mental health, moods, emotions, independence, relationships with parents and family life, financial resources, social support and friends, the school environment as well as social acceptance [8].

We examined the quality of life in the health of girls with menstrual disorders and girls without any disorders in health and development from both urban and rural areas. In the study we used the Polish version of the questionnaire to examine the quality of life related to health of children and adolescents KIDSCREEN-52.

Taking into account the variables such as patients age and area of residence, we found that girls with menstrual disorders aged 13-14 generally perceive their health as worse than girls without disorders in health and development. It should be noted that girls with menstrual disorders experience negative emotions more frequently and these situations threaten their sense of security with respect to health $(p<0.001)$.

The period of adolescence is a stage in a person's life when they make a transformation from a child into an adult. It is on the one hand the final phase of childhood, and on the other hand the beginning of adulthood. Over this short time radical changes occur, both physical and mental, which allow an individual to take on new tasks and social roles appropriate for an adult [13].

In the study of Mazur the respondents aged 8-18 assessed as worst the financial situation and school environment. Average indices of quality of life with respect to financial resources show the greatest diversity.

The highest index values were obtained for the dimension of social acceptance, which means rarely suffering from distress due to other pupils. Mental health measured according to the moods and emotions was assessed well, which means no enhancement of negative emotional states. The dimension of mental health relating to positive condition came out much worse.

Girls at puberty gave a worse evaluation of the quality of life in health with respect to physical health, moods and emotions, perception of oneself and independence, and a better evaluation of life quality in health with respect to contact with peers and the school environment.

The top rating was given by girls aged 13-14. Social acceptance was the exception, where the average indices of life quality increase with age, which is associated with less exposure to distress from other pupils [14]. Similarly, in our study, girls with menstrual disorders assessed their quality of life as worse with respect to the following dimensions: physical health, moods and emotions, perception of oneself and independence.

It should be noted that the studies were conducted in a healthy population and their results are consistent with the results of the research on a group of girls without any disorders in health and development. There is no reference material when it comes to results of KIDSCREEN-52 for girls with menstrual disorders.

The studies of Wojnarowska conducted with application of the questionnaire HBSC (Health Behaviour in School-aged Children) are known in the literature. Although the studies are devoted to the evaluation of health behaviours of young people, they contain a component of some assessment of dimensions of life quality in health, and therefore one can relate to them.

Wojnarowska found in her studies with the HBSC questionnaire that girls with menstrual disorders when compared to peer girls with typical or later maturation assessed their health as worse. They reported multiple ailments or negative emotional states and tried to lose weight despite not having excessive body weight. There were no differences between the groups in life satisfaction.

Early menstruating girls with menstrual disorders often smoked cigarettes regularly, abused alcohol, and had sexual intercourse. In that aspect early maturation is a risk factor for the health of girls $[6,14]$. From the analysis of our studies one can see that girls from the urban and rural environment had a negative perception of their health and well-being.

The deterioration of well-being resulted from menstrual disorders in adolescence. It can be concluded that it is a risk group with respect to disorders in mental health, in particular in the later years of life. Unfor- 
tunately, due to the use of two different research tools (HBSC - Wojnarowska; KIDSCREEN-52 - Mazur) the comparison of the results can be only general.

Children are our investment in the society of tomorrow. Their health and the way we raise them from the teenage years to maturity will affect the prosperity and stability of the countries in the European Region in the coming decades [15].

\section{Conclusions}

It was confirmed that the girls, aged 13-14, with menstrual disorders from the urban and rural areas obtain lower scores in all dimensions of quality of life related to health, compared to girls without such disorders in the light of the questionnaire KIDSCREEN-52. Prevalence of activities increasing the quality of life with particular emphasis on girls with menstrual disorders is likely to affect significantly the health and quality of life in the future. It may be important for therapeutic decisions to take into consideration the opinion of an individual on their well-being and quality of life in health, regardless of the clinical picture of the body. It may also be an indicator of unmonitored medical needs or other needs than health and as a result affect the health condition in adulthood and future generations.

\section{References}

1. Dziurowicz-Kozłowska A. Around the concept of life quality [Polish]. Psychology Life Quality 2002; 1: 77-79.

2. Harding L. Children's Quality of Life Assessments: a review of generic and health related quality of life measures completed by children and adolescents. Clin Psychol Psychother 2001; 8: 79-96.

3. WHOQOL Group. Development of the WHOQOL: rational and current status. Internal Journal of Mental Health 2003; 24-56.

4. Brzezińska A, Stolarska M, Zielińska J. A Sense of life quality in early, middle and late adulthood [Polish]. In: Tasks and social roles in adulthood. Appelt K, Wojciechowska J (eds.). The Humaniora Foundation Publisher, Poznań 2001; 103-126.

5. Pilch T, Leparczyk I. Social Pedagogy. Żak, Warsaw 2003, 61-70.

6. Woynarowska B. The rate of sexual maturation of girls and their health and the occurrence of risky behaviours. Polish Pediatrics (Warsaw) 2007; 82: 775-782.

7. Dutkiewicz W, Nowak-Starz G, Ciesla E. Standards and indicators of somatic and motoric development of children and youth in the Kielce Region. The Stachurski Publisher, Kielce 2004

8. Robitail S, Simeon MC, Erhart M, et al.; the Europen KIDSCREEN Group. Validation of the European proxy KIDSCREEN-52 pilot test health related quality of life questionnaire: first results. J Adolescent Health 2003; 5: 27-34.

9. The KIDSCREEN Group Europe. The KIDSCREEN Questionnaires. Quality of life questionnaires of children and adolescent. Handbook. Pabst Science Publishers, Lengerich Germany 2006.
10. Spilker B. Introduction. In: Quality of life and pharmacoeconomics in clinical trials. Spilker B (ed.). LippincottRaven, Philadelphia 2005.

11. Schipper H, Clinch J, Powell V. Quality of life studies: definitions and conceptual issues. In: Quality of life and pharmacoeconomics in clinical trials. Spilker B (ed.). Lippincott-Raven, Philadelphia 2007; 11-24.

12. Michel G, Bisegger C, Fuhr DC, Abel T; KIDSCREEN group. Age and gender differences in health-related quality of life of children and adolescents in Europe: a multilevel analysis. Quality Life Res 2009; 9: 1147-1157.

13. Zięba E, Nowak-Staż G, Karakiewicz B, et al. Somatic development of girls with menstrual disorders and no disoders in health and development with reference to selected weight and growth indicators. Medical Studies 2012; 25: 51-59.

14. Woynarowska B, Pułtorak M, Wojciechowska A. Health behaviours and the perception of their health by young people aged 11-16 in Poland. Physical Culture 2001; 7-8: 11-12.

15. Revens-Sieberer U, Gosch A, Rajmil L, et al. The European KIDSCREEN-52 quality-of-life measure for children and adolescents. Exp Rev Pharmacoeconom Outcom Res 2002; 5: 353-364.

\section{Address for correspondence:}

Ewa Zięba

Faculty of Health Sciences

Jan Kochanowski University

al. IX Wieków Kielc 19, 25-317 Kielce, Poland

E-mail: esz52@o2.pl 